\title{
Form finding of nexorades using the translations method
}

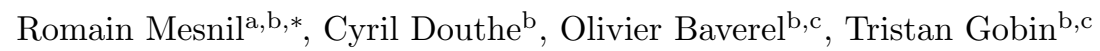 \\ ${ }^{a}$ École des Ponts ParisTech, Marne-La-Vallée, France \\ ${ }^{b}$ Laboratoire Navier, UMR 8205, École des Ponts, IFSTTAR, CNRS, UPE, Marne-La-Vallée, France \\ ${ }^{c}$ Université Paris-Est, École nationale supérieure d'architecture de Paris-Malaquais, Laboratoire Géometrie Structure Architecture, \\ Marne-La-Vallée, France
}

\begin{abstract}
This article proposes a new computational method for the form-finding of nexorades, also called reciprocal frames in the literature. The method is based on the translations of members forming the initial layout. It is shown that the two geometrical quantities defining nexorades - eccentricity and engagement length - depend linearly on the transformation parameters. The method introduced in this article is thus based on linear algebra, so that fitting problems can be formulated as simple quadratic optimisation problems under linear constraints. The proposed method is therefore fast, simple to implement, robust and can be applied to various grid patterns.

Furthemore, the proposed framework preserves planar facets. This paper proposes thus a new structural system where the nexorade is braced by planar facets. The feasibility of this structural system and of the computational framework introduced in this article is demonstrated by the fabrication of a $50 \mathrm{~m}^{2}$ timber pavilion.
\end{abstract}

Keywords: Constructive geometry, Space exploration, form-finding, reciprocal frame, reciprocal structure, nexorade, shell-nexorade hybrid, planar meshes, robotic timber construction, non-standard structures

\section{Introduction}

\subsection{Motivation}

Nexorades, also known as reciprocal frames [1, 2], are constituted of load-bearing members, which support each other along their span and not their extremities [3]. Their arrangement simplifies the construction of connection details, since only two members meet instead of four in quadrangular structures, or six in triangulated structures. They can be manufactured with robust and affordable techniques, like shown in Figure 1 while in comparison, a connection detail in free-form structure require complex manufacturing processes, due to higher node valence [4, 5].

Nexorades have been used since the middle age, for example by Villard DE HonneCOURT [7], or by Joseph ABEILle [8], both in France. The Zollinger lamella roof system, which was patented by Friedrich ZoLlinger, is another example of nexorade, particularly popular in Germany [9].

Despite their simplicity of assembly, nexorades are not widely used by engineers and architects. Two technical issues might explain this lack of popularity apart from cultural and circumstantial reasons. First, the form-finding of geometrical configurations of nexorades requires efficient non-linear solvers, which do not provide any certainty about their output [1]. Besides elementary cases,

\footnotetext{
* Corresponding author

E-mail: romain.mesnil@enpc.fr
}

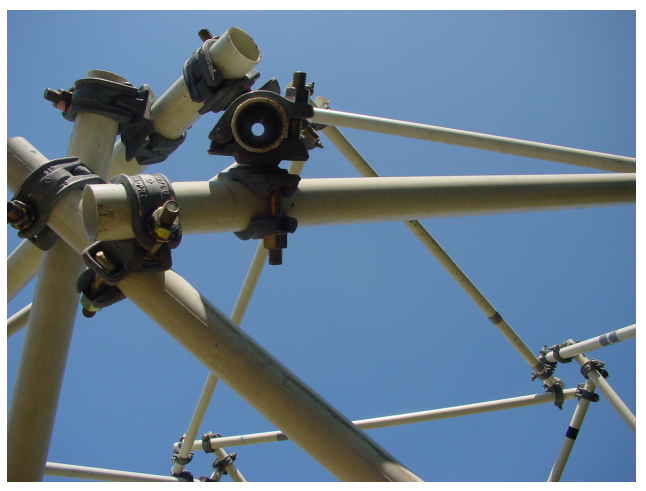

Figure 1: Simple two-valent connections in a nexorade (picture: [6])

like regular polyhedra $[10,6]$, cylindrical polyhedra or regular planar tilings, no theoretical result has been derived on the nature of the space of feasible nexorades. Second, nexorades are not as efficient as other structural systems mapping free-forms, like gridshells, because of their low node valence and low structural redundancy [11].

This paper is concerned with the form-finding of nexorades on doubly-curved surfaces with linear algebra. A restricted set of transformations is proposed to formulate simple geometrical quality metrics that can easily be minimised. Geometrical optimisation of nexorades within the proposed framework is discussed and an innovative bracing system is proposed to improve the structural behaviour. 


\subsection{Vocabulary}

Despite the fact that 'reciprocal frame' is the term generally used to describe this type of structure other terms would be more suitable as the term 'reciprocal frame' was originally used to describe only one node or a fan. The pioneer scientific work on these structures was done by BAVEREL et al. [1, 12], where it was first demonstrated that eccentricities and engagement length control the form of such structures. During this investigation, the term to describe these structures was 'Nexorade' (term proposed by Prof. H. Nooshin, nexor meaning link in latin) where most of the present vocabulary is coming from. Despite this argument on the name of this typology, many authors share a common vocabulary specific to the design of reciprocal frames or nexorades, which is recalled here $[1,12]$.

Consider first Figure 2: the displacement of several lines creates an opening called the engagement window. The assembly of members that constitutes the engagement window is referred to as a fan. The lengths of the engagement window (for example, the distance between $\mathbf{V}_{\mathbf{1}, \mathbf{i}}$ and $\mathbf{V}_{\mathbf{2}, \mathbf{e}}$ ) are called engagement length. The eccentricity is the distance between the axes of two attached members. It is a signed distance (it can be positive or negative with respect to a direction chosen by convention).
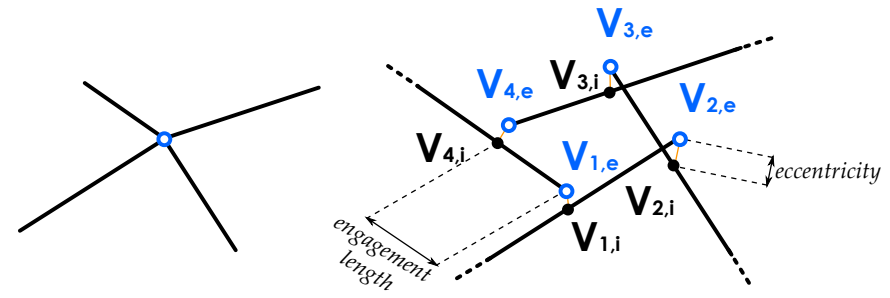

Figure 2: A fan obtained by disjunction of four concurrent lines.

Eccentricities are difficult to avoid when designing nexorades. Another parameter to consider is then the top/bottom disposition of the members. For a fan with $N$ in-going members, there are $2^{N}$ possible disposition (each contact can be oriented in a direction or the other). The simplest case of disposition is the so-called circular disposition, like on the left or middle of Figure 3. Noncircular disposition might be required for precise shapefitting problems [13]. Due to the vast number of possibilities, the top/bottom disposition is not a parameter directly controlled by the user in the present work, but will be the result of the optimisation procedure described in Section 4.

\subsection{Previous work}

Form-finding of nexorades. Nexorades are characterised by the interrelation between the different geometrical parameters. Their form-finding is an issue studied in numerous papers, and is often treated as an optimisation problem. BAVEREL [12] proposed to used genetic algorithms to perform the form-finding of nexorades. DouTHE and

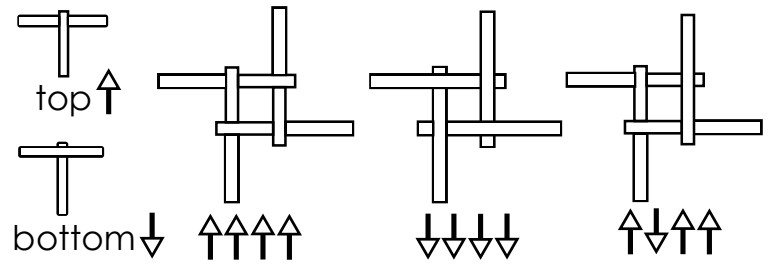

Figure 3: Some examples of top/bottom dispositions in a fan with four members, after [13] and [14].

BAVEREL proposed an adaptation of the dynamic relaxation (DR) [11] and also investigate the potential of this method for double layer systems [15]. The method is now implemented in several physics-based modelling tools [16]. Dynamic relaxation is a non-linear methods that can converge to local minima, but do not systematically converge towards the global minimum. Few papers deal with the analytical solution of the form-finding problem for nexorades: SENECHAL et al. studied the transformation of regular polyhedra by rotations [6], while BAVEREL studied analytically transformations by translations of regular polyhedra [12]. Finally, some papers approach form-finding of nexorades by setting the eccentricities as soft constraints and perform iterative least-square optimisation [14].

Structural behaviour of nexorades. Nexorades are rather unsusual objects for structural engineers. As beam structures, they can be analysed with the finite element or discrete element methods. For example, KoHLHAmmer and KoTNIK performed the analysis of flat nexorades by studying its decomposition in basic systems [17]. Douthe and BAVEREL analysed the structural response of nexorades under out-of-plane loads with dynamic relaxation, keeping the same design framework for the form-finding and structural analysis of nexorades [11]. They later explored the potential of double-layer neoxrades with the same analysis tool [15]. The bearing capacity of nexorades using glued connections has been evaluated by KOLHAMMER et al., the study demonstrates the interest to minimise eccentricities in timber nexorades [18]. This point is further discussed in sectino 4.2 .

Theoretical results are scarce, probably because of the complexity of the geometrical models and of the performance of modern parametric tools for modelling and analysis. GRECO et al. studied the out-of-plane response of nexorades with a homogenisation technique [19]. BROCATO showed that nexorades should map shapes that are optimised by minimising the axial forces, which is the opposite of what engineers tend to do with gridshells or shell structures [20]. This makes nexorades unusual objects for the majority of structural engineers, who are used to minimise bending and maximise axial forces in the structures they design. BRocATo and Mondardini also explored the potential of volumic nexorades, which allow to build flat stone vaults $[8,21]$. 
Linear subspace exploration and modelling. Linear space exploration is common in computer graphics, and can be applied to the modelling of surface discretizations that meet construction constraints. Poranne et al. proposed different methods to model meshes with planar facets with linear transformations of the nodal coordinates [22]. Applications to the modelling of gridshell structures have been proposed in $[23,24,25]$. A cartography of the dimensions of the linear subspaces is proposed for different structural patterns in [26].

Built projects. Nexorades are a mean to explore formal possibilities offered by elementary geometrical operations. They are often used for educational purpose, because they rely on rather simple detailing [27]. For example, the Plate Pavilion (Malta, 2014) uses plates rather than circular rods and explores the potential of offsets to create interlocking ${ }^{1}$. Full-scale architectural projects using nexorades are less common, and are often limited to temporary installations. Among recent projects, the KREOD pavilion demonstrates an interrelation between detailing and geometry $^{2}$. The Mount Rokko-Shidare Observatory is one of the largest nexorades built to date [28]. Finally, the roof over an archeological site in Bibracte, France is a good example offered by nexorades with zero eccentricity [29]. All the aforementioned projects are either covered with membranes, or uncovered, and provide thus little thermal or acoustical comfort. Nexorades have not benefited from recent advances in architectural geometry, where the fabrication of envelopes with planar or developable panels has been studied extensively [30]. Covering nexorades with planar panels could thus open new possibilities for their use, like for other structural systems [31].

\subsection{Contribution}

The design of nexorades mapping free-form surfaces requires fast and reliable tools. This article proposes to revisit the method of form-finding by translations initially presented in [12] and to present a novel framework for the form-finding of nexorades based on linear algebra, so that well-known results of linear algebra can be applied to determine the existence and uniqueness of solutions in optimisation problems. This article deals with two optimisation objectives: the fitting of engagement lengths, and the fitting of eccentricities. We focus on nexorades that minimise eccentricities rather than nexorades with constant eccentricities. This choice is governed by the technological choice for the practical realisation of connection (here a screwed T-joint, contrary to previous realisations using standard connectors), from a theoretical point of view, the method still apply for non-zero eccentricity.

Section 2 introduces notations and details the methodology used in this paper. Section 3 presents the construction of linear subspaces for the form-finding of nexorades.

\footnotetext{
${ }^{1}$ https ://design-milk.com/plate-pavilion-malta-design-week/
}

${ }^{2}$ http: //evolute.at/?p=2012_KREOD
Section 4 discusses numerical applications of geometrical optimisation with the construction subspaces. Section 5 introduces a novel structural system called shell-nexorade hybrid by the authors, and describes the geometrical formfinding of a timber pavilion implementing this concept.

\section{Methodology}

\subsection{Data structure}

Nexorades are constituted of members. In this article, and like in structural mechanics, members are idealised as lines, which follow their neutral axes. We focus on structures mapping 2-manifolds and consider the transformations of a mesh. The term edge in a mesh corresponds to the connection between two vertices, it is the combinatorial counterpart of the line that is drawn in the modeller. Figure 4 illustrates some transformation from a mesh pattern to a nexorade pattern. Notice the creation of engagement windows, in blue on the Figure.

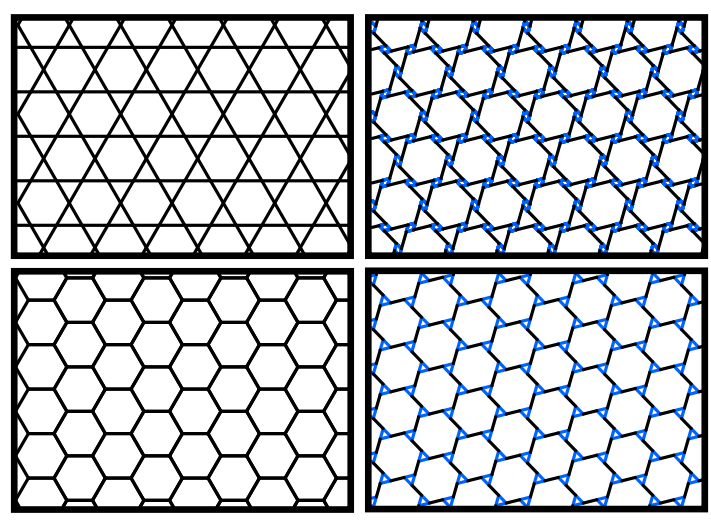

Figure 4: Nexorades (right) obtained from mesh patterns (left)

Figure 2 shows the creation of a fan from the disjunction of four concurrent lines. Usually, there is some eccentricity between the members: to each end node, we associate the closest point on the attached line. Each line has two ends: we store their coordinates in a column-vector $\mathbf{X}_{\mathbf{e}}$.

$$
\left\{\begin{aligned}
\mathbf{X}_{\mathbf{e}, 3 \mathbf{i}}: & x \text { coordinate of node } \# \mathrm{i} \\
\mathbf{X}_{\mathbf{e}, 3 \mathbf{3}+\mathbf{1}}: & y \text { coordinate of node } \# \mathrm{i} \\
\mathbf{X}_{\mathbf{e}, \mathbf{3} \mathbf{i}+\mathbf{2}}: & z \text { coordinate of node } \# \mathrm{i}
\end{aligned}\right.
$$

It is also necessary to store information on proximities between lines. We call intermediate node the node corresponding to an end node on an adjacent member, as shown in Figure 2. The coordinates of the intermediate nodes are stored in a column-vector $\mathbf{X}_{\mathbf{i}}$ of same size as $\mathbf{X}_{\mathbf{e}}$.

The notation $\mathbf{C}$ is used for the edge-node connectivity matrix. It is a matrix with $3 n_{n}$ rows and $3 n_{e}$ columns, where $n_{n}$ and $n_{e}$ denote the number of nodes and edges (and lines or members) respectively. Note that we define the number of nodes as the number of end nodes, so that $n_{n}=2 n_{e}$. 
$\forall \alpha \in[0,2], C_{3 i+\alpha, 3 j+\alpha}=\left\{\begin{array}{r}1 \text { if node } \# \mathrm{i} \text { belong to edge } \# \mathrm{j} \\ 0 \text { else }\end{array}\right.$

Let us write $\mathbf{X}$ and $\mathbf{X}^{\prime}$ the coordinates of the nodes before and after the transformation. They are related by a linear equation:

$$
\mathbf{X}^{\prime}=\mathbf{C} \cdot \mathbf{T}+\mathbf{X}
$$

A member always has at most two intermediate nodes, regardless of the used tiling pattern. The data-structure is therefore adapted to arbitrary connectivities and is not limited to uniform tilings, a limitation of some techniques in existing literature.

\subsection{Notations}

In the followings of this article, bolded letters describe either vectors or matrices. The notations for the number of nodes, edges, and faces are detailed in Table 1. Greek letters are used as weighting parameters between different optimisation functionals, and do not refer to eccentricity, contrarily to some notations found in previous literature.

\begin{tabular}{|c|c|}
\hline Description & Notation \\
\hline Number of edges & $n_{e}$ \\
\hline Number of inner edges & $n_{i}$ \\
\hline Number of boundary edges & $n_{b}$ \\
\hline Number of nodes & $n_{n}$ \\
\hline Number of faces & $n_{F}$ \\
\hline Eccentricity & $e$ \\
\hline Engagement length & $l$ \\
\hline
\end{tabular}

Table 1: Notations for the scalar values

Vectors and matrices usually refer to the linear space of translation of all the edges. We use specific notation in $\mathbb{R}^{3}$. The translation of the $i^{t h}$ edge is written $\mathbf{T}_{, \mathbf{i}}$. The subscript $t$ is used to describe target eccentricities or engagement lengths in fitting problems (see Table 2).

\subsection{Parallel transformations}

The geometry of nexorades can be generated using two principles: the rotation of the members or the translation of the members, or a combination of both, as proposed in [12]. This article proposes to focus on translations, which can be seen on Figure 5. There are still two ways to create a fan from converging lines. One can imagine pulling the ends of each bar and look at the rotation of the fan. If the fan rotates clockwise, the fan is said to be rightward, else it is leftward [1, 11].

\subsection{Boundaries of nexorades}

Boundaries of nexorades must be treated separately because they yield fans with different valence and they have less interaction with other structural members. The working hypothesis in this article is shown in Figure 6. The designer sets first a mesh as input (left of the Figure). The members that are on the boundary, i.e. the members that belong to one face only, are then deleted. The data structure is then constructed from the network in the middle of Figure 6. An example of possible nexorade arising from our computational framework is shown on the right of Figure 6.
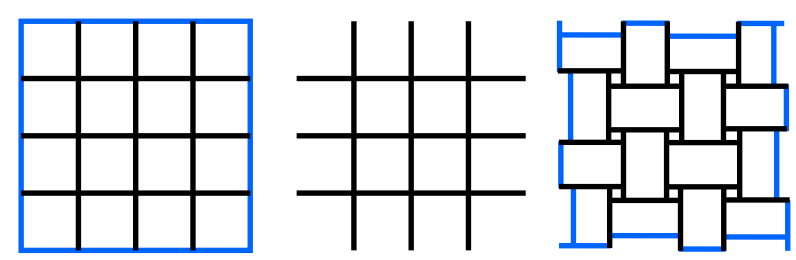

Figure 6: Treatment of boundaries in this article: members on the mesh boundary (in blue) are not taken into account in the formfinding procedure.

In practical applications where free-edge must be treated separately, additional members can be added afterwards. The blue lines on the right of Figure 6 correspond to members that are added after the form-finding procedure. Because those lines are only supported on the form-found geometry (on black lines on the Figure), their eccentricity or engagement lengths can be found by solving uncoupled problems, which is considerably simpler than solving a system of coupled equations, the main difficulty in the form-finding of nexorades.

\section{Construction of linear subspace for nexorades}

The key contribution of this article is to describe the design space for nexorades by geometrical transformations rather than nodal coordinates, like previously done in the literature. This approach is inspired from the form-finding of polyhedral meshes by Vaxman [32]. We propose elementary proof that, in nexorades obtained by translation, eccentricity and engagement length depend linearly on the transformation parameters. We start from the relations between two attached lines and then assemble the linear constraints in a matrix form. Building upon these results, it is possible to create a linear subspace for shape exploration with nexorades.

\subsection{Eccentricity and distance between two lines}

The eccentricity is the distance between two attached members. This section recalls the distance between two lines, as represented in Figure 7 and studies its variations when the two lines are translated. We consider thus two 


\begin{tabular}{|c|c|c|}
\hline Description & Notation & Dimension \\
\hline Null-vector of $\mathbb{R}^{n}$ & $\mathbf{0}_{\mathbf{n}}$ & $n \times 1$ \\
\hline Initial coordinates of the end nodes & $\mathbf{X}_{\mathbf{e}}$ & $3 n_{n} \times 1$ \\
\hline Final coordinates of the end nodes & $\mathbf{X}_{\mathbf{e}}^{\prime}$ & $3 n_{n} \times 1$ \\
\hline Initial coordinates of the intermediate nodes & $\mathbf{X}_{\mathbf{i}}$ & $3 n_{n} \times 1$ \\
\hline Final coordinates of the intermediate nodes & $\mathbf{X}_{\mathbf{i}}^{\prime}$ & $3 n_{n} \times 1$ \\
\hline Translation vector & $\mathbf{T}$ & $3 n_{n} \times 1$ \\
\hline Translation of edge $\# i$ & $\mathbf{T}_{\mathbf{i}}$ & $3 \times 1$ \\
\hline Edge-node connectivity matrix & $\mathbf{C}$ & $3 n_{e} \times 3 n_{n}$ \\
\hline Eccentricities & $\mathbf{E}$ & $\left(2 n_{i}+n_{b}\right) \times 1$ \\
\hline Target eccentricities & $\mathbf{E}_{\mathbf{t}}$ & $\left(2 n_{i}+n_{b}\right) \times 1$ \\
\hline Engagement length & $\mathbf{L}$ & $\left(2 n_{i}+n_{b}\right) \times 1$ \\
\hline Target engagement length & $\mathbf{L}_{\mathbf{t}}$ & $\left(2 n_{i}+n_{b}\right) \times 1$ \\
\hline Eccentricity matrix & $\mathbf{M}_{\mathbf{E}}$ & $\left(2 n_{i}+n_{b}\right) \times 3 n_{e}$ \\
\hline Engagement length matrix & $\mathbf{M}_{\mathbf{L}}$ & $\left(2 n_{i}+n_{b}\right) \times 3 n_{e}$ \\
\hline
\end{tabular}

Table 2: Notations for the vectors and matrices
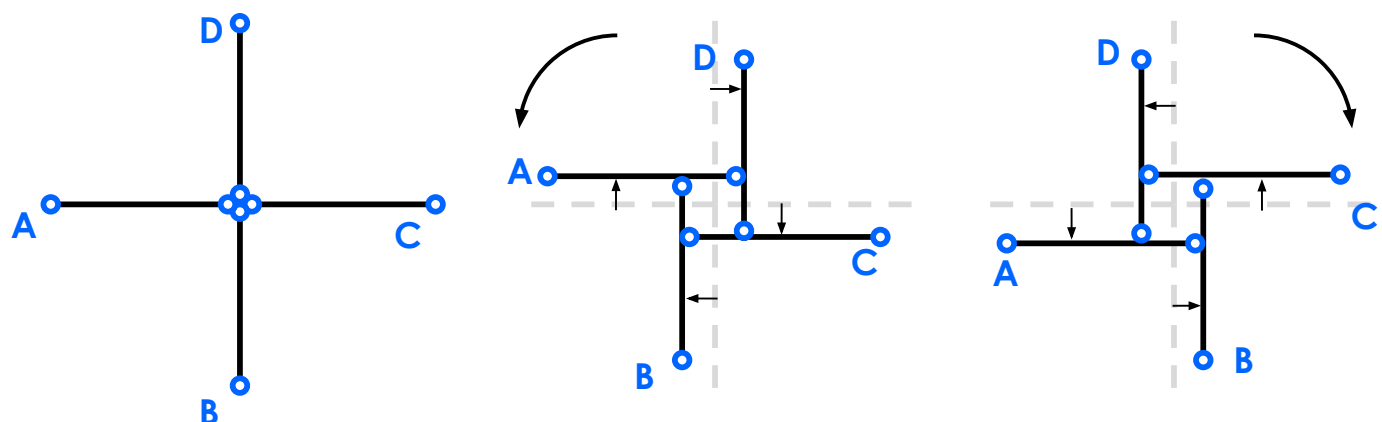

Figure 5: Possible fan orientations (left: initial configuration, middle: leftward fan, right: rightward fan).

lines $\left(\mathcal{D}_{0}\right)$ and $\left(\mathcal{D}_{1}\right)$ going through the points $\mathbf{M}_{\mathbf{0}}$ and $\mathbf{M}_{\mathbf{1}}$ respectively. Their guiding vectors are written $\mathbf{t}_{\mathbf{0}}$ and $\mathbf{t}_{\mathbf{1}}$.

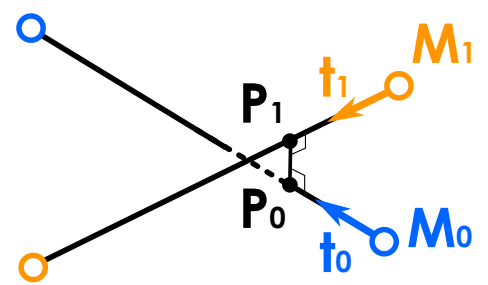

Figure 7: Notations for the evaluation of the distance between two lines.

The signed distance between the lines, i.e. the eccentricity, is simply given by equation (4).

$$
d\left(\mathcal{D}_{0}, \mathcal{D}_{1}\right)=\frac{\operatorname{det}\left(\mathbf{M}_{\mathbf{1}}-\mathbf{M}_{\mathbf{0}}, \mathbf{t}_{\mathbf{0}}, \mathbf{t}_{\mathbf{1}}\right)}{\left\|\mathbf{t}_{\mathbf{0}} \wedge \mathbf{t}_{\mathbf{1}}\right\|}
$$

Notice that the orientation of the members appears at the numerator and denominator of equation (4). Modifying the orientations of the members yields non-linear equations, which require iterative solvers. In the following, we study the change of this distance when the lines $\mathcal{D}_{i}$ are translated: their guiding vectors do not change.

$$
\mathcal{D}^{\prime}{ }_{i}=\mathcal{D}_{i}+\mathbf{T}_{\mathbf{i}}
$$

Since the vectors $\mathbf{t}_{\mathbf{0}}$ and $\mathbf{t}_{\mathbf{1}}$ are not changed by the translation, equation (4) becomes:

$$
d\left(\mathcal{D}^{\prime}{ }_{0}, \mathcal{D}^{\prime}{ }_{1}\right)=\frac{\operatorname{det}\left(\mathbf{M}_{\mathbf{1}}-\mathbf{M}_{\mathbf{0}}+\mathbf{T}_{\mathbf{1}}-\mathbf{T}_{\mathbf{0}}, \mathbf{t}_{\mathbf{0}}, \mathbf{t}_{\mathbf{1}}\right)}{\left\|\mathbf{t}_{\mathbf{0}} \wedge \mathbf{t}_{\mathbf{1}}\right\|}
$$

The determinant is 3-linear, so that we can expand it:

$$
d\left(\mathcal{D}^{\prime}{ }_{0}, \mathcal{D}^{\prime}{ }_{1}\right)=d\left(\mathcal{D}_{0}, \mathcal{D}_{1}\right)+\frac{\operatorname{det}\left(\mathbf{T}_{\mathbf{1}}-\mathbf{T}_{\mathbf{0}}, \mathbf{t}_{\mathbf{0}}, \mathbf{t}_{\mathbf{1}}\right)}{\left\|\mathbf{t}_{\mathbf{0}} \wedge \mathbf{t}_{\mathbf{1}}\right\|}
$$

In the present study, the lines $\left(\mathcal{D}_{0}\right)$ and $\left(\mathcal{D}_{1}\right)$ are originally concurrent, so that the first term is equal to zero. After further expansion, equation (7) becomes:

$$
e=d\left(\mathcal{D}^{\prime}{ }_{0}, \mathcal{D}^{\prime}{ }_{1}\right)=\frac{\operatorname{det}\left(\mathbf{T}_{\mathbf{1}}, \mathbf{t}_{\mathbf{0}}, \mathbf{t}_{\mathbf{1}}\right)-\operatorname{det}\left(\mathbf{T}_{\mathbf{0}}, \mathbf{t}_{\mathbf{0}}, \mathbf{t}_{\mathbf{1}}\right)}{\left\|\mathbf{t}_{\mathbf{0}} \wedge \mathbf{t}_{\mathbf{1}}\right\|}
$$

The eccentricity depends thus linearly on $\mathbf{T}_{\mathbf{0}}$ and $\mathbf{T}_{\mathbf{1}}$.

\subsection{Engagement length}

In a fan, like the one shown in Figure 2, each member is in relation with two other members: loosely speaking each 
member is supported by another member and supports a third member. A simplified representation of the interactions between three members of a fan is shown in Figure 8: the points $\mathbf{P}_{\mathbf{i}}$ are the ones minimising the distances between the members neutral axes. The signed distance between $\mathbf{P}_{\mathbf{1}}$ and $\mathbf{P}_{\mathbf{2}}$ is the engagement length $l$. We introduce the notation $\mathbf{t}$ for a guiding vector of the line pointing away from the fan.

$$
l=\left(\mathbf{P}_{\mathbf{2}}-\mathbf{P}_{\mathbf{1}}\right) \cdot \frac{\mathbf{t}_{\mathbf{1}}}{\left\|\mathbf{t}_{\mathbf{1}}\right\|}
$$

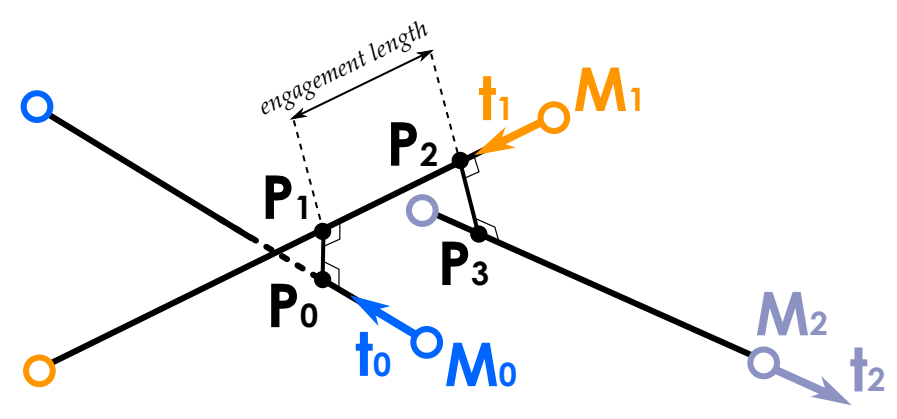

Figure 8: Notations for the evaluation of the engagement length.

One must thus compute the position of the points $\mathbf{P}_{\mathbf{i}}$. The closest point to line 1 on line 0 is written $\mathbf{P}_{\mathbf{0}}$, as shown in Figure 8. Its position is found by solving a simple least square problem. The derivation is straight forward and yields equation (10), with the assumption that $\mathbf{t}_{\mathbf{1}} \neq \mathbf{t}_{\mathbf{0}}$, which is always verified in practice for nexorades. We write $\Delta=\mathbf{M}_{0}-\mathbf{M}_{1}$.

$$
\mathbf{P}_{\mathbf{0}}=\mathbf{M}_{\mathbf{0}}+\frac{\left\|\mathbf{t}_{\mathbf{1}}\right\|^{2} \cdot\left(\boldsymbol{\Delta} \cdot \mathbf{t}_{\mathbf{0}}\right)-\left(\mathbf{t}_{\mathbf{0}} \cdot \mathbf{t}_{\mathbf{1}}\right)\left(\boldsymbol{\Delta} \cdot \mathbf{t}_{\mathbf{1}}\right)}{\left\|\mathbf{t}_{\mathbf{1}}-\mathbf{t}_{\mathbf{0}}\right\|^{2}} \cdot \mathbf{t}_{\mathbf{0}}
$$

Like previously, we study the evolution of $\mathbf{P}_{\mathbf{0}}$ when translating the lines $\mathcal{D}_{0}$ and $\mathcal{D}_{1}$. In order to simplify notations, we introduce the function $f$, which is defined as follows:

$$
\begin{aligned}
& f\left(\mathbf{T}_{\mathbf{0}}, \mathbf{T}_{\mathbf{1}}, \mathbf{t}_{\mathbf{0}}, \mathbf{t}_{\mathbf{1}}\right)= \\
& \frac{\left\|\mathbf{t}_{\mathbf{1}}\right\|^{2} \cdot\left(\left(\mathbf{T}_{\mathbf{0}}-\mathbf{T}_{\mathbf{1}}\right) \cdot \mathbf{t}_{\mathbf{0}}\right)-\left(\mathbf{t}_{\mathbf{0}} \cdot \mathbf{t}_{\mathbf{1}}\right)\left(\left(\mathbf{T}_{\mathbf{0}}-\mathbf{T}_{\mathbf{1}}\right) \cdot \mathbf{t}_{\mathbf{1}}\right)}{\left\|\mathbf{t}_{\mathbf{1}}-\mathbf{t}_{\mathbf{0}}\right\|^{2}} \cdot \mathbf{t}_{\mathbf{0}}
\end{aligned}
$$

The evolution of the closest point of $\mathcal{D}_{1}$ on $\mathcal{D}_{0}$ with respect to the translations $\mathbf{T}_{\mathbf{0}}$ and $\mathbf{T}_{\mathbf{1}}$ is then given by:

$$
\mathbf{P}_{\mathbf{0}}^{\prime}=\mathbf{P}_{\mathbf{0}}+f\left(\mathbf{T}_{\mathbf{0}}, \mathbf{T}_{\mathbf{1}}, \mathbf{t}_{\mathbf{0}}, \mathbf{t}_{\mathbf{1}}\right)
$$

In the form-finding problem, $\mathbf{t}_{\mathbf{0}}$ and $\mathbf{t}_{\mathbf{1}}$ are fixed. It results that $f$ is a linear function of $\mathbf{T}_{\mathbf{0}}$ and $\mathbf{T}_{\mathbf{1}}$. The development of equation (9) can be made by using this property. We shall also notice, that in our framework, the lines initially meet because the initial input is a mesh. We can thus write following equation:

$$
\left\{\begin{array}{l}
\mathbf{P}_{\mathbf{1}}=\mathbf{M}_{1}+f\left(\mathbf{T}_{1}, \mathbf{T}_{\mathbf{0}}, \mathbf{t}_{\mathbf{1}}, \mathbf{t}_{\mathbf{0}}\right) \\
\mathbf{P}_{\mathbf{2}}=\mathbf{M}_{\mathbf{1}}+f\left(\mathbf{T}_{\mathbf{1}}, \mathbf{T}_{\mathbf{2}}, \mathbf{t}_{\mathbf{1}}, \mathbf{t}_{\mathbf{2}}\right)
\end{array}\right.
$$

It follows that:

$$
l=\left(f\left(\mathbf{T}_{\mathbf{1}}, \mathbf{T}_{\mathbf{2}}, \mathbf{t}_{\mathbf{1}}, \mathbf{t}_{\mathbf{2}}\right)-f\left(\mathbf{T}_{\mathbf{1}}, \mathbf{T}_{\mathbf{0}}, \mathbf{t}_{\mathbf{1}}, \mathbf{t}_{\mathbf{0}}\right)\right) \cdot \frac{\mathbf{t}}{\|\mathbf{t}\|}
$$

The engagement length depends also linearly on the $\mathbf{T}_{\mathbf{0}}, \mathbf{T}_{\mathbf{1}}$ and $\mathbf{T}_{\mathbf{2}}$.

\subsection{Orientation of the fans and assembly}

The remarks made on the relations between two lines can be generalised to the whole structure. The key observation is that, as shown in Figure 1, there are only two-valent connections in nexorades. The linear equations described in the previous part can thus be assembled in a matrix form.

To do so, one needs to define the orientation of the fans. The present paper deals with meshes on manifolds, so that the half-edge data structure can be used to retrieve informations about nodal orientation. An open-source .NET library [33] that implements the half-edge data structure is used in the numerical applications in this article. The half-edge data structure is based on the construction of half-edges which are associated to a unique face, as shown in Figure 9. For a given node, there is a list of in-going halfedges (respectively out-going half-edges). The end-user only sets his or her preferences for the fan orientation. If the fan is leftwards, then the vector $\mathbf{t}$ used in equation (14) corresponds to the orientation of the ingoing half-edges, the vectors should be reversed for rightwards nodes. This choice is automated thanks to the half-edge data structure, including for configurations with alternation of rightwards or lefwards fans with complex topologies.

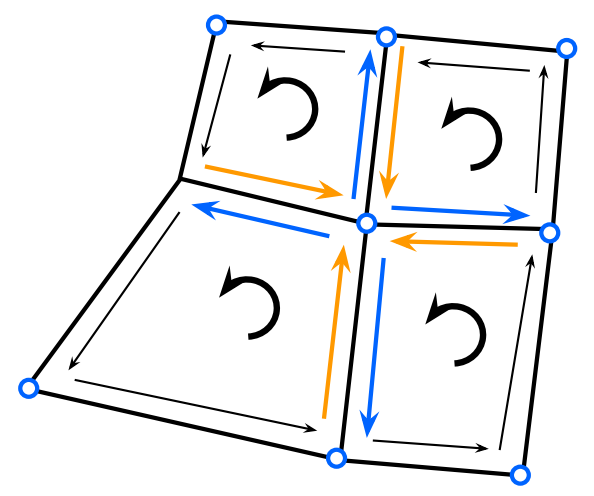

Figure 9: The half-edge data structure on a quadrangular mesh: ingoing (in orange) and outgoing (in blue) half-edges for the central node.

Equation (7) can thus be assembled in a matrix form written in equation (15). Each inner edge is attached to two fans at each of its extremities, whereas boundary edges are only attached to one fan, there are thus $\left(2 n_{i}+n_{b}\right)$ eccentricities measures, and the matrix $\mathbf{M}_{\mathbf{E}}$ has $\left(2 n_{i}+n_{b}\right)$ rows.

$$
\mathbf{E}^{\prime}=\mathbf{M}_{\mathbf{E}} \cdot \mathbf{T}
$$

Likewise, the engagement lengths depend linearly on the translation parameters: we introduce the notation $\mathbf{M}_{\mathbf{L}}$ for 
the matrix that assembles linear constraints. Like $\mathbf{M}_{\mathbf{E}}$, $\mathbf{M}_{\mathbf{L}}$ has $\left(2 n_{i}+n_{b}\right)$ rows.

$$
\mathbf{L}^{\prime}=\mathbf{M}_{\mathbf{L}} \cdot \mathbf{T}
$$

Both $\mathbf{M}_{\mathbf{E}}$ and $\mathbf{M}_{\mathbf{L}}$ are sparse, because low connectivity of nexorades. To be more precise, the number of non-zero terms in the matrices is of $6\left(2 n_{i}+n_{b}\right)$ and $9\left(2 n_{i}+n_{b}\right)$ for the eccentricity and engagement length respectively (for each row, six translation components are involved in equation (8) and nine in equation (14)). The proportion of non-zero terms in $\mathbf{M}_{\mathbf{E}}$ and $\mathbf{M}_{\mathbf{L}}$ is thus $\frac{1}{n_{e}}$ and $\frac{3}{2 n_{e}}$ respectively. The number of members being typically comprised between 100 and 1000, there is at most $1 \%$ of non-zero terms in $\mathbf{M}_{\mathbf{E}}$ and $\mathbf{M}_{\mathbf{L}}$.

\subsection{Filtering of non-degenerate translations}

Some translations do not affect the eccentricites and engagement lengths and should be filtered out in order to avoid ill-conditioned systems. For example, the translation of a line along its guiding vector does not change the eccentricity or the engagement length, they are referred to as 'degenerate solutions' in the following of this article. We impose thus the $n_{e}$ independent constraints defined by equation (17).

$$
\forall i \in\left[1, n_{e}\right], \mathbf{T}_{\mathbf{i}} \wedge \mathbf{t}_{\mathbf{i}}=\mathbf{0}
$$

This constraint is computed for each edge and assembled afterwards. By doing so, we compute $n_{e}$ kernels of $3 \times 3$ matrices (complexity of $\mathcal{O}\left(n_{e}\right)$ ) instead of a single kernel of a $3 n_{e} \times 3 n_{e}$ matrix (complexity of $\left.\mathcal{O}\left(n_{e}^{3}\right)\right)$. We guarantee thus the computational performance of the method.

Among possible transformations, it is possible to have rigid body modes where all the members are assigned the same translation. Those rigid body modes should also be discarded, since they do not change eccentricities, nor engagement lengths. This removes three degrees of freedom. These constraints reduce thus the dimension of the design space from $3 n_{e}$ degrees of freedom to $2 n_{e}-3$ degrees of freedom. Those considerations lead to the construction of a matrix $\mathbf{N}$ with $3 n_{e}$ rows and $2 n_{e}-3$ columns. The design space of nexorades by edge translation is thus defined as follows:

$$
\mathbf{T}=\mathbf{N} \mathbf{T}^{\prime}
$$

The vector $\mathbf{T}^{\prime}$ is a vector of $\mathbb{R}^{2 n_{e}-3}$ that corresponds to the transformation parameters with non-degenerate translations. Like $\mathbf{M}_{\mathbf{E}}$ and $\mathbf{M}_{\mathbf{L}}$, the matrix $\mathbf{N}$ is sparse (with zeros density above $95 \%$ in the treated examples of this paper).

\subsection{Null-space and geometrical interpretation}

In numerical applications, the computation of the nullspace of the matrices $\mathbf{M}_{\mathbf{E}}$ and $\mathbf{M}_{\mathbf{L}}$ is necessary. The nullspace of $\mathbf{M}_{\mathbf{E}}$ defines transformations with parallel edges with no eccentricities, while the null-space of $\mathbf{M}_{\mathbf{L}}$ defines transformations with parallel edges with no engagement length. The intersection of both null-spaces defines therefore transformations with parallel edges with no eccentricities and no engagement lengths. In other words, such transformations map a mesh to another mesh with the same connectivity, where all the corresponding edges are parallel. An example of such transformation is shown in Figure 10.
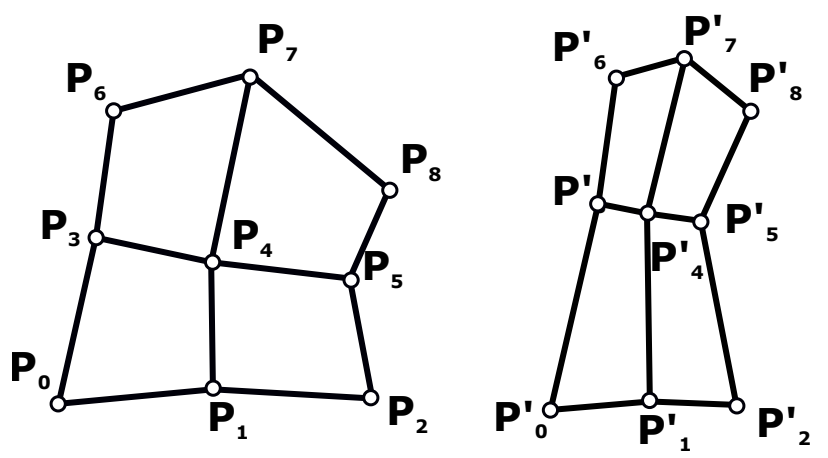

Figure 10: Two quadrilateral meshes related by a discrete Combescure transformation, all their respective edges are parallel.

Such transformations have already been studied in previous literature and are known as discrete Combescure transformations [34]. PORANne et al. showed how to construct the linear space of discrete Combescure transformations by assembling linear constraints computed on each mesh faces [22]. These trasnformations are found in various contexts, for example in the study of equilibrium of thin shell structures [35]. They can also be used to generate torsion-free support layouts or structures with high node congruence [4]. An estimation of the dimension of the space of discrete Combescure transformations is given in [34] and recalled in equation (19).

$$
\operatorname{dim}\left(\operatorname{Ker}\left(\mathbf{M}_{\mathbf{E}}\right) \cap \operatorname{Ker}\left(\mathbf{M}_{\mathbf{L}}\right)\right) \simeq n_{e}-2 n_{F}+3
$$

This equation should be understood as follows: the only parameter accessible is the length of each edge, but one can choose only $(n-2)$ independent length parameters for each face for the parallelity condition to be satisfied. There are thus two constraints for each face. The number 3 correponds to the three translations in space that leave edge length invariant.

We are now able to write non-degenerate transformations by translations of a given mesh, which are not discrete Combescure transformations. They represent the practical design space for nexorades by translations. The next section proposes to study the potential of this new design space and the influence of the different design parameters or objectives with different numerical experiments.

\section{Numerical application: form-finding by optimi- sation}

Designers aim at controlling two geometrical quantities when designing nexorades: the eccentricity and the 
engagement length. This section discusses the possibility to use quadratic optimisation to control these two quantities simultaneously.

\subsection{Geometrical optimisation of nexorades}

Based on equation (15), the error metric for the fitting of eccentricities can be written as a quadratic functional of $\mathbf{T}$ and $\mathbf{E}_{\mathbf{t}}$, where $\mathbf{E}_{\mathbf{t}}$ is a vector of target eccentricities (constant for example in $[11,15]$ ).

$$
\mathcal{F}_{E}=\left(\mathbf{M}_{\mathbf{E}} \cdot \mathbf{T}-\mathbf{E}_{\mathbf{t}}\right)^{T} \cdot\left(\mathbf{M}_{\mathbf{E}} \cdot \mathbf{T}-\mathbf{E}_{\mathbf{t}}\right)
$$

A similar error metric can be constructed for the fitting of engagement lengths $\mathbf{L}_{\mathbf{t}}$ with equation (16):

$$
\mathcal{F}_{L}=\left(\mathbf{M}_{\mathbf{L}} \cdot \mathbf{T}-\mathbf{L}_{\mathbf{t}}\right)^{T} \cdot\left(\mathbf{M}_{\mathbf{L}} \cdot \mathbf{T}-\mathbf{L}_{\mathbf{t}}\right)
$$

In practice, the designers aim at simultaneously optimising the engagement length and eccentricities. We propose thus a combined optimisation function written as a weighted sum of the previous objective functions. Notice that no term corresponding to the proximity to the reference surface is used, this choice and its practical implications are discussed in section 4.4. The examples discussed in the following of this paper demonstrate that the introduction of a proximity functional is indeed unnecessar, and ensured indirectly through the low values of $\mathbf{E}_{\mathbf{t}}$ and the uniformity of values of $\mathbf{L}_{\mathbf{t}}$.

$$
\mathcal{F}(\lambda, \mathbf{T})=\lambda \mathcal{F}_{L}(\mathbf{T})+(1-\lambda) \mathcal{F}_{E}(\mathbf{T})
$$

The scalar $\lambda$ is a weighting factor between the two objectives: it is comprised between 0 (priority to the optimisation of eccentricities) and 1 (priority to the optimisation of engagement lengths). The optimisation problem is written as a constrained optimization problem:

$$
\min _{\mathbf{T}=\mathbf{N} \cdot \mathbf{T}^{\prime}} \lambda \mathcal{F}_{L}(\mathbf{T})+(1-\lambda) \mathcal{F}_{E}(\mathbf{T})
$$

We have seen in Section 3.5 that the intersections of the null-spaces of $\mathbf{M}_{\mathbf{E}}$ and $\mathbf{M}_{\mathbf{L}}$ is not empty, and correspond to Combescure transformations. This implies that the problem is under-constrained, since we can notice that:

$$
\left\{\begin{array}{l}
\forall \mathbf{T} \in \mathbb{R}^{3 n_{e}}, \forall \mathbf{T}^{*} \in \operatorname{Ker}\left(\mathbf{M}_{\mathbf{E}}\right), \mathcal{F}_{E}\left(\mathbf{T}+\mathbf{T}^{*}\right)=\mathcal{F}_{E}(\mathbf{T}) \\
\forall \mathbf{T} \in \mathbb{R}^{3 n_{e}}, \forall \mathbf{T}^{*} \in \operatorname{Ker}\left(\mathbf{M}_{\mathbf{L}}\right), \mathcal{F}_{L}\left(\mathbf{T}+\mathbf{T}^{*}\right)=\mathcal{F}_{L}(\mathbf{T})
\end{array}\right.
$$

Therefore, we further constrain the problem by constructing the set of discrete Combescure transformations. Like in [22], we construct the set of Combescure transformation by a matrix $\mathbf{N}_{\mathbf{1}}$ so that $\mathbf{N}_{\mathbf{1}} \mathbf{T}^{\prime}=\mathbf{0}$ by assembling the constraints for each face. In the optimisation problem, we restrict $\mathbf{T}^{\prime}$ to be in the column-space of $\mathbf{N}_{\mathbf{1}}$. This column space is computed with the linear algebra library Math.NET Numerics [36]. We compute thus a full-rank matrix $\mathbf{N}_{\mathbf{2}}$, which contains an orthonormal basis of the column space of $\mathbf{N}_{\mathbf{1}}$.

$$
\mathbf{T}^{\prime}=\mathbf{N}_{\mathbf{2}} \mathbf{T}^{\prime \prime}
$$

We can thus reformulate the optimisation problem as follows:

$$
\min _{\mathbf{T}=\mathbf{N} \cdot \mathbf{N}_{\mathbf{2}} \cdot \mathbf{T}^{\prime \prime}} \lambda \mathcal{F}_{L}(\mathbf{T})+(1-\lambda) \mathcal{F}_{E}(\mathbf{T})
$$

This is a quadratic optimisation problem under linear constraints, which can be solved with a closed-form formula. The derivation of the formula is classical and recalled in the Appendix.

\subsection{Choice of target eccentricities}

Nexorades with constant eccentricity are particularly simple to construct, since one unique connection detail can be used as in the fan depicted in Figure 1. Possible target eccentricities include all the vectors with constant eccentricity $e$, either positive or negative.

$$
\forall i, \mathbf{E}_{\mathbf{t} i}= \pm e
$$

The number of possible target eccentricities with this practical constraint is thus of $2^{2 n_{i}+n_{b}}$. As the number of members increases, the difficulty for the designer is thus to choose properly the signs in equation (27).

In the followings of this paper, eccentricities are optimised towards zero, mainly because of the application to the wooden pavilion shown in Section 5. The proposed detailing shown in this article does not rely on half-lapped joints or scaffolding connectors, which are the most common details found in the literature. Half-lapped joints significantly decrease the static height of the members and weakens thus the structure, while scaffolding connectors are only suited for cylindrical members, which are not commonly found for timber. Two simple alternatives are glued connections and end-grain screws: glued connections have been proposed in [37], while this paper explores endgrain screws. With this kind of connection, the smaller the eccentricities, the larger the contact surface between members and therefore the higher the level-arm between screws. Minimising eccentricities improves thus the structural behaviour and eases constructability.

$$
\mathbf{E}_{\mathbf{T}}=\mathbf{0}_{\mathbf{2} \mathbf{n}_{\mathbf{i}}+\mathbf{n}_{\mathbf{b}}}
$$

The eccentricity is an objective of the optimisation, and is therefore not treated as a hard constraint, but rather as a soft constraint. This is contrary to the point of view of BAVEREL and NoOsHIN, who considered that eccentricity was a technological constraint [1]. The treatement of the equations is however simplified, and this strategy has already been adopted in previous articles [14].

The results of the form-finding with zero-eccentricity can be used as a hint for the form-finding with non-zero eccentricity. Once the first step of the form-finding with zero-eccentricity is achieved, the designer can retrieve the signs of the eccentricities E: this gives thus a preferred top/bottom arrangement for the members. The idea of choosing the top-bottom arrangement from a preliminary step rather than using it as a design parameter is present in previous form-finding techniques $[11,14]$. If one considers 
building with cylindrical members, the optimal radius of the rods can be found by solving a linear programming problem.

\subsection{Choice of target engagement lengths}

Engagement lengths can be optimised towards a target length, constant throughout the mesh, or to a length that is proportional to the initial member length. In order to clarify the discussion in the followings, we will choose one type of optimisation and target constant eccentricities and engagement lengths. Engagement lengths are optimised towards a constant length for all fans.

$$
\mathbf{L}_{\mathbf{t}}=L_{T} \cdot \mathbf{1}_{\mathbf{2} \mathbf{n}_{\mathbf{i}}+\mathbf{n}_{\mathbf{b}}}
$$

where $\mathbf{1}_{\mathbf{2} \mathbf{n}_{\mathbf{i}}+\mathbf{n}_{\mathbf{b}}}$ is a vector of dimension $2 n_{i}+n_{b}$ containing only 1's. The target engagement length $L_{T}$ can be defined relatively to the average of the member lengths $l_{\text {mean }}$ in the initial configuration. We may introduce in this case the scalar $\alpha$, which takes values between 0 and 0.5 . Large values of $\alpha$ correspond to large engagement lengths.

$$
L_{T}=\alpha \cdot l_{\text {mean }}
$$

The influence of the parameter $\alpha$ will be investigated in the next section.

\subsection{Influence of the connectivity}

Architects and engineers can choose different fan orientations and create structures with different behaviours. Although the framework proposed in this article can be generalised to arbitrary mesh connectivities, we focus here on nexorades obtained as transformations of quadrilateral meshes, which are ubiquituous in free-form architecture.

We discuss here the choice of two strategies: one where all the fans have the same orientation (leftwards for example) and one where two adjacent fans have opposite orientations. The latter one is possible only when the nodes are two-colorable, which restricts the meshing strategies of a surface. The choice of either pattern has an influence on the structural performance of the nexorade, as discussed in [11] and [12]. We propose here to discuss the relation between the choice of the pattern and the performance of the optimisation presented in this Section.

Figure 11 shows a nexorade found on an ellipsoid meshed by its lines of curvature. It features closed curves and two singularities of type 'lemon'. The engagement length ratio is $\alpha=0.1$, a typical value for nexorades.

The mesh has to be adapted to transform the two quads attached to a singularity of valence 2 into two triangles, as shown in Figure 12, because a fan with two members cannot be closed. Notice that, by modifying the connectivity, the nodes on the right of Figure 12 are not two-colorable anymore. The two nodes belonging to the triangles have the same orientation.

Figures $13 \mathrm{a}$ and $13 \mathrm{~b}$ show the relative performance of the two fan orientation strategies. The error is measured as follows:

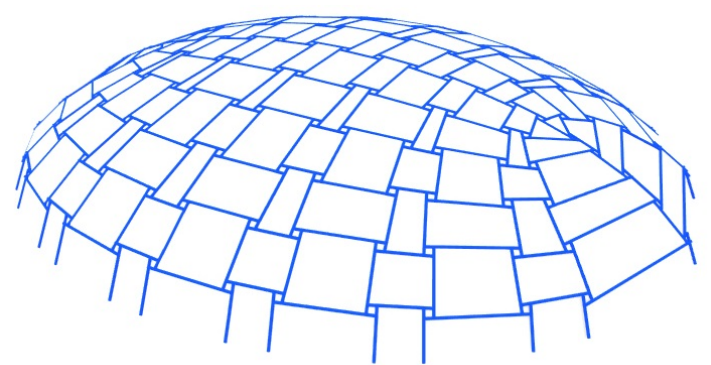

Figure 11: Nexorade obtained from the lines of curvature of an ellipsoid $(\alpha=0.1)$

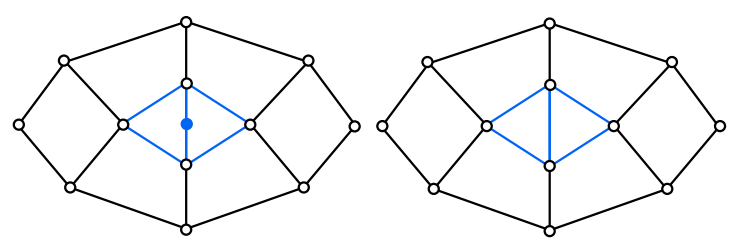

Figure 12: Connectivity change around the umbilical point of the ellipsoid. Initial configuration with two quads (left) and modified connectivity (right).

$$
\left\{\begin{array}{lr}
l_{\max }= & \max \left(\left\|L_{i}-L_{t}\right\|\right) \\
e_{\max }= & \max \left(\left\|E_{i}\right\|\right)
\end{array}\right.
$$

Figure 13 shows the errors normalised with the target engagement length for the nexorade depicted in Figure 11. In practical applications, members usually have a slenderness (length-over-height ratio) smaller than 10. Considering that the target engagement length is $10 \%$ of the average member length, we should thus recall that designers aim at a relative error smaller than 1 in Figure 13b. Remarkably, the normalised errors proposed in Figure 13 do not depend on the value of $\alpha$ : indeed it can be shown (see Appendix) that the optimal eccentricities and engagement lengths depend linearly on $\alpha$ when the target eccentricty is the null vector, so that normalising them with the target engagement length yields a constant value.

The two alternation strategies have similar performances when optimising for engagement length at higher values of $\lambda$, as shown in Figure 13a. The maximal error is located at the singularity, where the member arrangement is the most complex. The relative difference between the maximal errors for the two strategies is of $4 \%$ for $\lambda=0.98$. Notice however that the two strategies yield very different results when optimising for eccentricities, i.e. $\lambda$ is small. It can be seen in Figure 13b that the orientation strategy has a different influence on the eccentricities. The eccentricity obtained with the alternated orientations is two times lower. Alternating fan orientations seems thus to provide better results in terms of geometrical fitting.

This is visually confirmed by Figure 14, which shows the best-fitting nexorade of an input mesh with two alternation strategies. When the fans all have the same orientation, the shape seems to rotate. Notice in particular the 


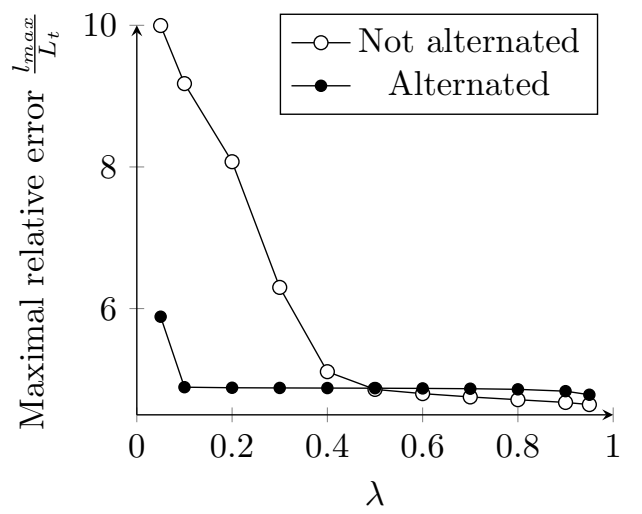

(a) Engagement lengths.

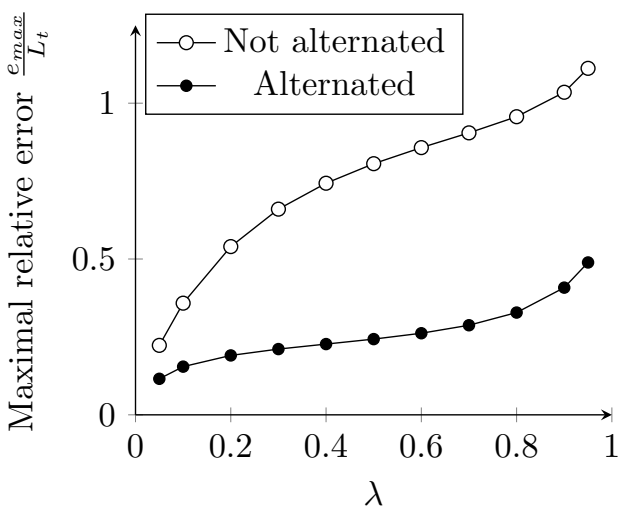

(b) Eccentricities.

Figure 13: Comparison of the influence of the alternation strategy on the maximal error made on engagement lengths and eccentricities for the ellipsoid.

axis of symmetry, which is originally horizontal in Figure 14:

- with the alternated pattern, the points of the axis do not move much but the top and bottom lose their symmetry with respect to the horizontal line. Because the shape has initially two axes of symmetry, the resulting nexorade still has a symmetry of rotation. Choosing two different fan orientations (by changing leftwards fans into rightwards fans and vice-versa) yields the same shape up to a reflection in the case of Figure 14.

- with the non-alternated pattern, the points of the axis move away from the horizontal line. The shape has no symmetry. Choosing two different fan orientations (only leftwards or only rightwards) yields two different shapes here. The mesh is however much more uniform in this case.

These symmetries properties are inherent to the alternation strategies and can be referred to as chirality in material science community. A chiral structure has a nonsuperimposable mirror image of itself. The alternate translation strategy yields an anti-tetra-chiral pattern while the non-alternated one is a tetra-chiral pattern [38].

In the following of this article, we consider only the alternated pattern, since it provides better results in the fitting problems. The potential of this strategy can be illustrated through Figures 15, which show another application for a shape with a complex topology, meshed initially with four singularities. To the best of the author's knowledge, no previous work in the literature studied patterns with several singularities.

The resulting shape has several closed loops and singularities. It should be noted that the overall shape is visually preserved by the transformation, although proximity to the initial shape is not included in the objective function. This is confirmed by studying the distance between the fan centroid (defined as the barycentre of the ends of the lines of the members), and the its initial position before transformation. This distance is on average a fraction of the engagement length $\left(1 / 6^{t h}\right.$ of the target engagement length in Figure 15). Moreover, the average distance of the fan centroid to the reference mesh is only $1 / 40^{t h}$ of the target engagement length in Figure 15, which means that the vertices move in the osculating plane of the reference surface. The proximity of the nexorade to the initial surface is due to the filtering of rigid body modes and Combescure transformations in the optimisation formulation.

\subsection{Influence of optimisation parameter}

The designer aiming at optimising engagement lengths and eccentricities must find the compromise between the two objectives, which is determined by the value of $\lambda$ in equation (22). Some comments can be made on the influence of its value on the output of the optimisation. We propose to illustrate this with another shape with a complex topology. Figure 16 displays three nexorades that result from the combined optimisation problem of equation (22). There are 1320 inner edges and 176 boundary edges.

The example of Figure 16 is tested for 23 different values of $\lambda$. We recall that $\mathbf{E}$ and $\mathbf{L}$ are proportional to $\alpha$. Figure 17 represents the influence of the value of $\lambda$ on the maximal error made on eccentricity and engagement length for the initial geometry depicted in Figure 16. With small values of $\lambda$, the combined optimisation sets preference on engagement length fitting.

Notice finally that $e_{\max }$ tends towards zero when $\lambda$ is small. It is logical, since $\mathbf{T}=\mathbf{0}$ is an obvious minimiser of $\mathcal{F}_{E}$ when $\mathbf{E}_{\mathbf{T}}=\mathbf{0}$, but it is not a minimiser of $\mathcal{F}_{L}$. This remark only holds for $\mathbf{E}_{\mathbf{T}}=\mathbf{0}$ and because we filter Combescure transformations and rigid body modes (which are also obvious minimisers of $\mathcal{F}_{E}$ when $\left.\mathbf{E}_{\mathbf{T}}=\mathbf{0}\right)$. 


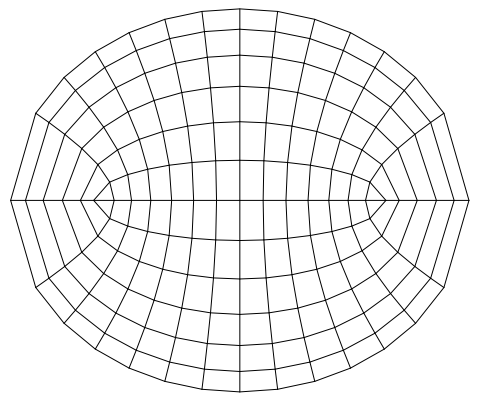

(a) Reference mesh

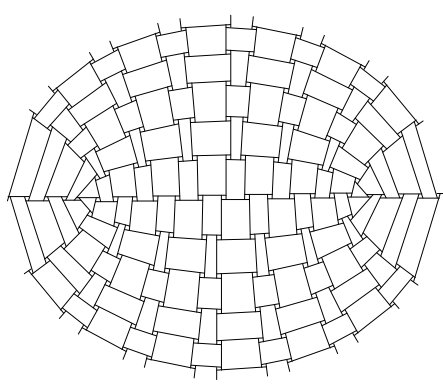

(b) Alternated pattern

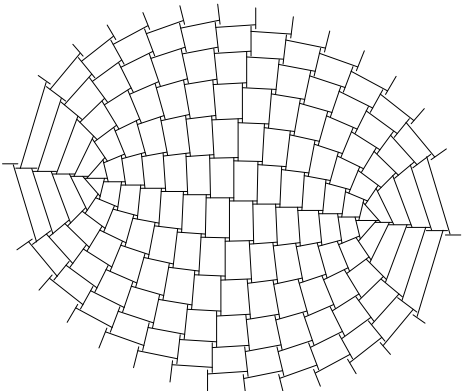

(c) Non-alternated pattern

Figure 14: Comparison of the alternate and non-alternate translations for the fitting of an ellipsoid $(\alpha=0.1, \lambda=0.8)$.

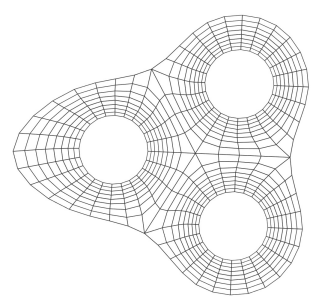

(a) Top view.

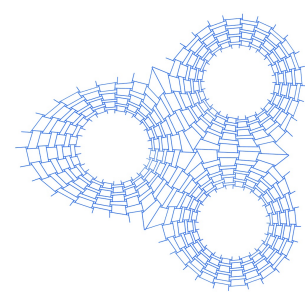

(c) Top view.

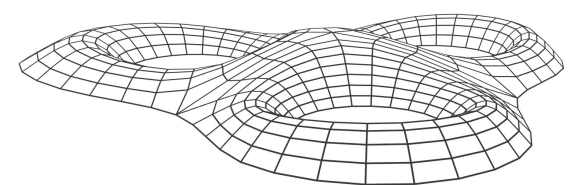

(b) Perspective.

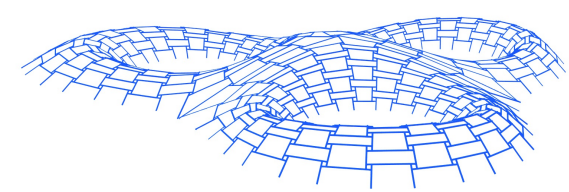

(d) Perspective.
Figure 15: Reference geometry with three nodes of even valence on the boundaries and resulting nexorades with alternate translation $(\alpha=0.1, \lambda=0.85)$.

\section{Discussion on practical applications}

With the influence of the different design parameters on the output of the form finding procedure having been discussed, we can focus on practical applications of the framework proposed in the present article.

\subsection{Shell-nexorade hybrid}

The framework proposed for the morphogenesis of nexorades is based upon translation of the edges of a mesh. Coplanarity of lines is preserved by translation of either lines. This fact is used in surfaces of translations, which have been proposed by Schlaich and Schober to generate gridshells covered by planar facets [39]. For nexorades, it means that starting with a reference mesh with planar facets and applying the translation method yields a nexorade covered with planar facets. The planar facets can be used to brace the nexorade, if mechanically connected to the members. We call shell-nexorade hybrid the resulting structural system, because it behaves as a shell.
The literature on mesh planarisation for architecture is rich, and the designers are not restricted to triangular facets only. Indeed, surfaces can be covered with planar quadrangular or hexagonal facets, for example if they are meshed along their lines of curvature [40, 41], like in Figure 11. The form finding technique proposed in this article can thus build upon the rich literature on polyhedral meshes to generate shell-nexorade hybrids.

\subsection{Offsets}

By definition, connections in nexorades are two-valent, meaning that only two members meet at a connection. Systems with low valence generally have many possible offset with planar members and torsion-free nodes. If one views nexorades as meshes, the connection is three-valent, and there is an alignment constraint between two lines, as illustrated in Figure 18, where the vertices are marked as white circles. Three valent systems necessarily admit constant face offsets, which allows for the use of plates of constant thickness to cover and brace the structure.

\subsection{Design and construction of a shell-nexorade hybrid: the 2017 Build'in Pavilion}

Digital manufacturing technologies enable new possibilities for nexorades. In particular, non-uniform eccentricities can be dealt with custom robotised fabrication [37]. A timber pavilion, shown in Figure 19 has been fabricated to illustrate the potential of the proposed structural system. It spans $6.5 \mathrm{~m}$ and cover $50 \mathrm{~m}^{2}$ with a structural depth of 14 centimeters. The 102 members are braced by more than sixty planar quadrangular timber panels.

Figure 20 shows the different iterations for the form finding of the pavilion. First, a reference geometry is defined based on the site constraints. Then, a fitting problem is solved with the marionette technique in order to find a PQ-mesh in the vicinity of the reference surface [23, 24]. The translation technique proposed in the previous sections is then applied: the objective is to minimize eccentricities and to have uniform engagement lengths. The optimal fitting problem and translation technique are both based on the solution of one linear system and can thus be 


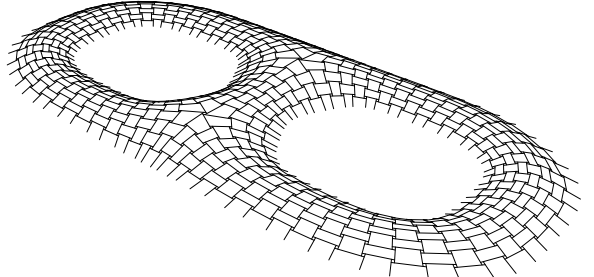

(a) $\alpha=0.359, \mathcal{F}_{E}=0.201, \mathcal{F}_{L}=0.7797$

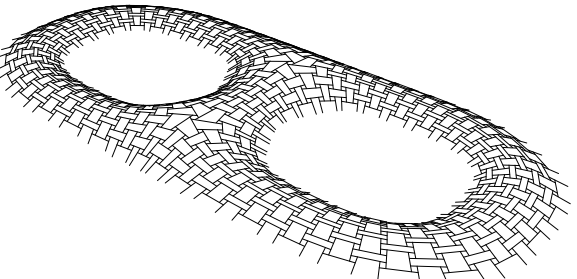

(b) $\alpha=0.718, \mathcal{F}_{E}=0.805, \mathcal{F}_{L}=3.1187$

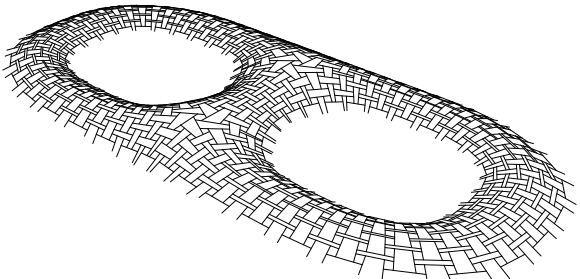

(c) $\alpha=1.077, \mathcal{F}_{E}=1.812, \mathcal{F}_{L}=7.017$

Figure 16: Three nexorades with a complex topology optimised with $\lambda=0.95$

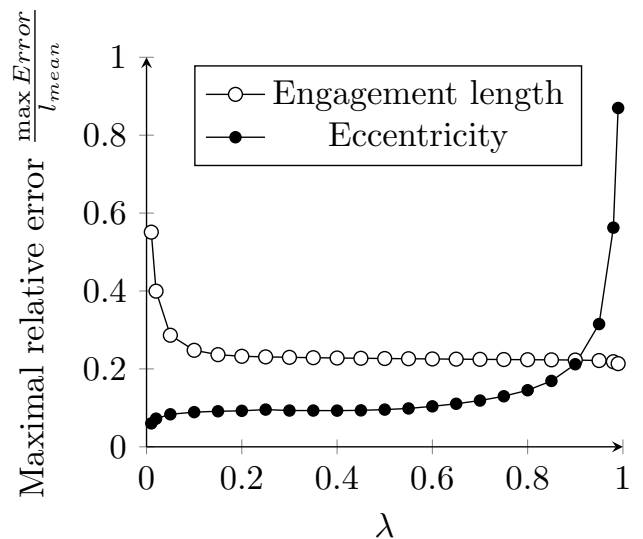

Figure 17: Influence of the value of $\lambda$ on relative errors of eccentricities and engagement lengths, $\alpha=0.5385$.
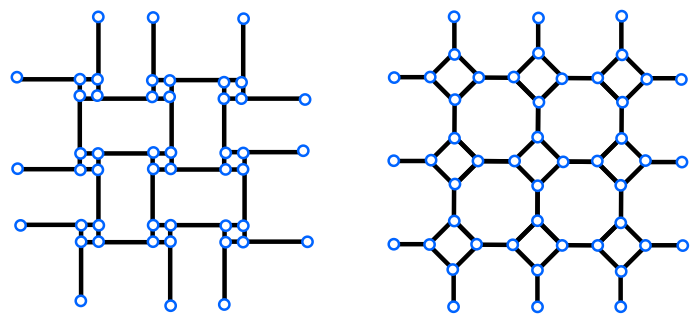

Figure 18: Nexorade pattern viewed as mesh patterns: topological equivalence between the alternated quad nexorade pattern and the truncated square pattern.

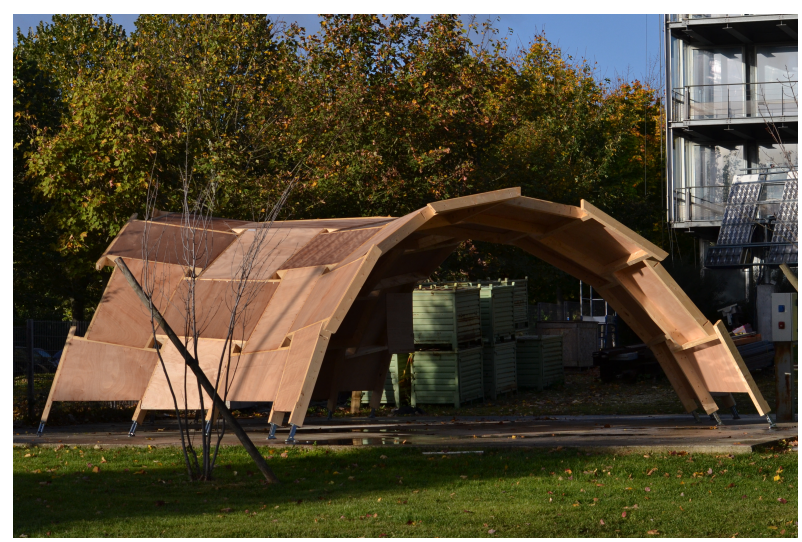

Figure 19: Timber pavilion using the principle of hybrid nexorades (copyright: Romain Mesnil).

conducted in real-time. Several iterations between engagement length and reference geometry have been performed in order to satisfy both mechanical and fabrication constraints.

Figure 21 shows the T-junction between two members. Screws are used because they are relatively simple to put on site and provide a good mechanical behaviour. Guiding holes have been drilled with robotic arms in order to ease the on-site assembly. Three screws are following the neutral axis of the incoming member (guiding vector: $\mathbf{e}_{\mathbf{x}}$ ). The rotation along the weak axis of the incoming member (guiding vector: $\mathbf{e}_{\mathbf{z}}$ on the picture) are allowed by the connection detail, but the two other rotations (out-of plane bending and member torsion) are restricted. This makes the structure highly redundant and stable even without the panels.

Figure 22 shows a detail of the attachement between the panels and members. The engagement window is not covered by a panel. This image should be compared to Figure 2, which shows a fan. It seems here that there is no eccentricity, but the members were milled with two collaborating 6-axes robots in order to avoid the direct exposure of the fibers and to erase the offset of members top surface. The higher the eccentricity, the more timber is removed from the cross-section and the weaker the section. This 


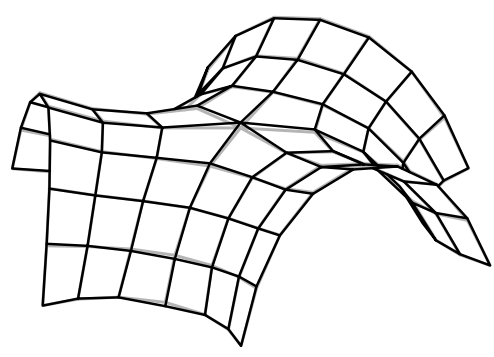

(a) Reference geometry (gray) and bestfit PQ-mesh with a marionette mesh (black).

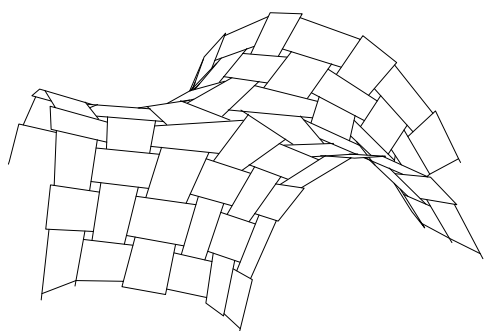

(b) Optimised nexorade.

Figure 20: Two steps of the morphogenesis of the timber pavilion.

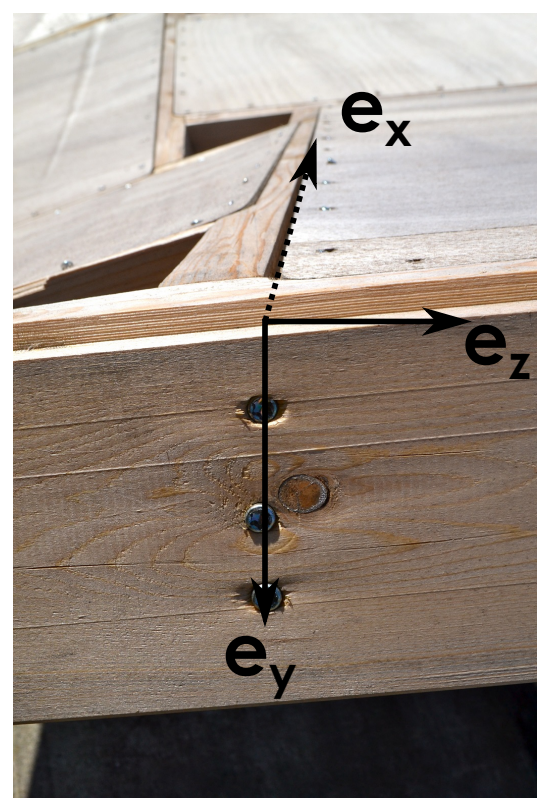

Figure 21: Attachment between members, (copyright: Romain Mesnil).

explains why this article focuses on optimisation towards zero eccentricity.

\subsection{Benefits of shell-nexorade hybrids}

The detailed structural behaviour of shell-nexorade hybrids is beyond the scope of this paper, but it can be illustrated with a simple comparative study of the pavilion shown in this section with or without plates used as a bracing system. We considered different load cases: self-weight, symmetrical snow load, non-symmetrical wind

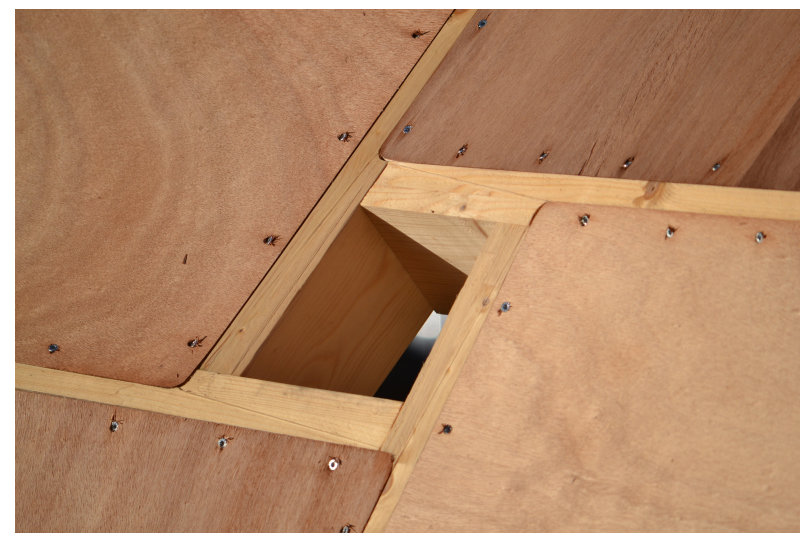

Figure 22: Attachment between flat panels and members, (copyright: Romain Mesnil).

loads and eccidental loads according to the Eurocode. The results of our study show that the shell-nexorade hybrid outperforms the unbraced nexorade, with much smaller forces applied to the connection details, as illustrated in Figure 23. The average bending moment in the connection is divided by 8 , shear and axial forces are also significantly lower in the shell-nexorade hybrid.

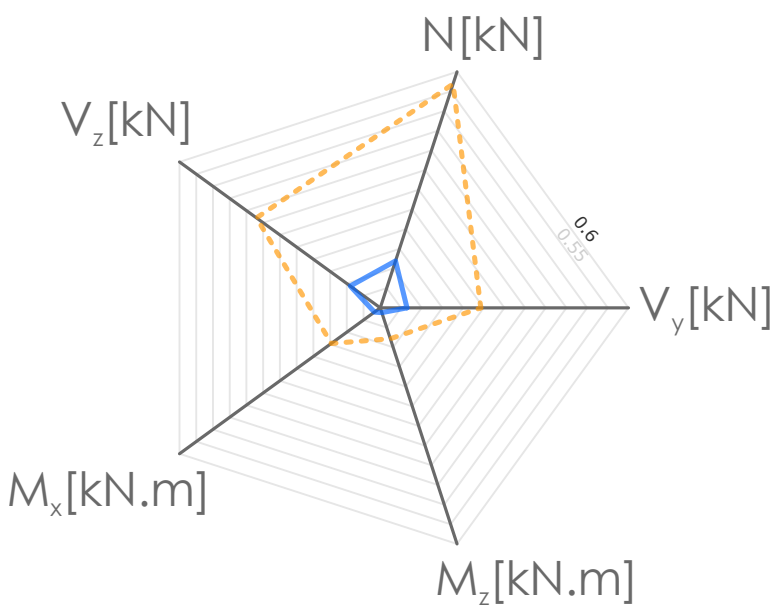

Figure 23: Average amplitude of forces and moment in connections for shell-nexorade hybrid (thick blue) and nexorade without panels (dotted orange).

The planar plates stiffen significantly the structure: the maximal displacement under wind load is divided by 12 , with a mass increase of approximately 30\%. The innovative bracing system based on the translation technique developed in this paper and used in the pavilion is thus proved to be highly efficient and opens new perspectives of applications for nexorades.

The difference between the two models can be explained by the coupling between axial forces and in-plane bending in unbraced nexorades. On the left of Figure 25, it appears that the members are subject to a rotation, especially on the central fan. On the right, this deformation mode does not exist, and the structure is subject to an 


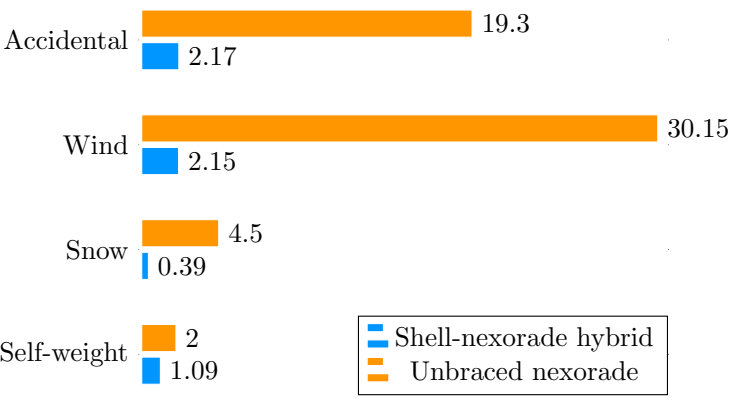

Figure 24: Maximal displacements ( $\mathrm{mm}$ ) for different load cases.

out-of-plane deformation (amplification of deformation is identical for both pictures).
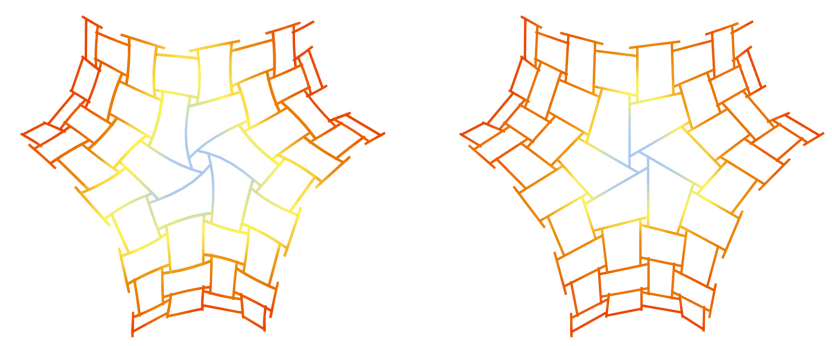

Figure 25: Displacement under uniform snow load of $0.2 \mathrm{kPa}$, seen from top: left unbraced structure, right: shell-nexorade hybrid (displacements are magnified 100 times, and panels are removed for better comparison on the right).

\section{Conclusion}

This article proposed a linear formulation for the formfinding of nexorades by translation. This simplifies the optimisation for the fitting of engagement lengths or eccentricities, since it reduces to quadratic optimisation under linear constraint. Although the proposed method is suited to various patterns, a special focus has been made on nexorades derived from quadrilateral meshes. Future work can include application to other structural patterns, which have been proven to be structurally efficient and constructible [42]. The examples treated in the present paper consider eccentricity as an optimisation objective rather than a hard constraint. The method could also be coupled with existing form-finding methods, like dynamic relaxation, in order to fully satisfy constraints of eccentricies used in classical nexorades.

We also proved the invariance of the solutions by discrete Combescure transformations, which are naturally used to generate double layer structures. The proposed methodology could thus be extended to double-layer nexorades. Furthermore, we have demonstrated that the translation method preserves facet planarity, which opens possibilities for bracing nexorades with planar structural components.
This article proposed a new structural system called shell-nexorade hybrid. Its structural potential has been demonstrated by the construction of a large-scale timber pavilion. The fabrication of a full-scale pavilion allowed for some insight about the scalibility of hybrid nexorades. Fabrication and sites constraints required looping between the form-finding procedure, structural analysis and geometry assessment. The reliability of the form-finding method proposed in this paper was key to the success of the design procedure.

The method used in this article takes inspiration of recent advances in computer graphics to solve a constructability problem. The identification construction constraints and their invariants by geometrical transformations for atomic elements of a structure significantly simplifies the form-finding of the whole structure. The form finding of nexorades deals with alignment constraints and is thus likely to rely on geometrical transformations that play a particular role in line geometry. The methodology proposed in this paper could thus be applied to nexorades with other transformations, like projective transformations, or to other structural systems.

\section{Acknowledgements}

The timber pavilion presented in Section 5 is the first prototype built in the context of a teaching and research agreement in the Build'in platform of the Co-Innovation Lab of l'Ecole des Ponts. It could not have been built without the support of our industrial partners: ABB France, Hal Robotics Ltd, Simonin SAS, Schunk France and Würth France.

The authors warmly thank the people who took part in the design and construction of the timber pavilion: Leo Demont, Hocine Delmi, Nicolas Ducoulombier, Pierre Margerit, Xavier Tellier, Mathieu Tintillier, Loic Simonin, Stephen Connord, Thibault Mandalaz, Jean-Francois Caron, Sébastien Gervillers, Vianney Loing, Philippe Hannequart, Romain Boulaud, Nicolas Leduc, Paul Carneau and the students who took part to the construction of the pavilion: Aurélien Beck, Mathilde Bellec, Alban Berrubé, Mirko Antonio Borzone, Ignacio Canela Gomà, Louise Chatelain-Lacam, Enrique Corres Sojo, Maxime Dupont, Laure Duval, Pau Figueras Cadenet, Quentin Glevarec, Brieuc Graillot, Yukiho Kamata, Anne Levionnois, Ricardo Maia Avelino, Sébastien Maitenaz, Matteo Migliari, Nicolas Montagne, Bastian Ninino, Rafael Henrique Ortiz Hazarian, David Pastor Moreno, Pedro Henrique Pedrosa Torres, Jérôme Pitance, Thiago Ramos Musman, Amine Souiki, Agnieszka Tatarczak, Frederico Vaz Guilherme, Nóra Veres Lia, Ana Clara Vianna Da Costa e Faria and Sonia Zerhouni.

The authors also thank Laurent Hauswirth for fruitful discussions on discrete differential geometry, and Alain Ehrlacher for his kind support. 


\section{References}

[1] O. Baverel, H. Nooshin, Y. Kuroiwa, G. Parke, Nexorades, International Journal of Space Structures 15 (2) (2000) 155-159. doi:https://doi.org/10.1260/0266351001495053.

[2] O. Baverel, O. P. Larsen, A review of woven structures with focus on reciprocal systems-nexorades, International Journal of Space Structures 26 (4) (2011) 281-288. doi:https://doi.org/ 10.1260/0266-3511.26.4.281.

[3] A. Pugnale, M. Sassone, Structural reciprocity: Critical overview and promising research/design issues, Nexus Network Journal 16 (1) (2014) 9-35. doi:https://doi.org/10.1007/ s00004-014-0174-z.

[4] R. Mesnil, C. Douthe, O. Baverel, B. Léger, J.-F. Caron, Isogonal moulding surfaces: a family of shapes for high node congruence in free-form structures, Automation in Construction 59 (2015) 38-47. doi:https://doi.org/10.1016/j.autcon. 2015. 07.009.

[5] J. Knippers, T. Helbig, Recent developments in the design of glazed grid shells, International Journal of Space Structures 24 (2) (2009) 111-126. doi:https://doi.org/10.1260/ 026635109789043205

[6] B. Sénéchal, C. Douthe, O. Baverel, Analytical investigations on elementary nexorades, International Journal of Space Structures 26 (4) (2011) 313-320. doi:https://doi.org/10.1260/ 0266-3511.26.4.313.

[7] T. Bowie, The sketchbook of Villard de Honnecourt, Greenwood Press, 1960.

[8] M. Brocato, L. Mondardini, A new type of stone dome based on abeilles bond, International Journal of Solids and Structures 49 (13) (2012) 1786-1801. doi:https://doi.org/10.1016/j. ijsolstr.2012.03.036.

[9] F. Zollinger, Means op manufacturing spa, uS Patent 1,483,037 (Feb. 5 1924).

[10] O. Baverel, H. Nooshin, Nexorades based on regular polyhedra, Nexus Network Journal 9 (2) (2007) 281-298. doi:https:// doi.org/10.1007/s00004-007-0043-0.

[11] C. Douthe, O. Baverel, Design of nexorades or reciprocal frame systems with the dynamic relaxation method, Computers \& Structures 87 (21) (2009) 1296-1307. doi:https://doi.org/ 10.1016/j.compstruc. 2009.06.011.

[12] O. L. Baverel, Nexorades: a family of interwoven space structures, Ph.D. thesis, University of Surrey (2000).

[13] D. Parigi, P. H. Kirkegaard, M. Sassone, Hybrid optimization in the design of reciprocal structures, in: International Association for Shell and Spatial Structures - Asian Pacific Conference on Shell and Spatial Structures 2012, The International Association for Shell and Spacial Structures, 2012.

[14] P. Song, C.-W. Fu, P. Goswami, J. Zheng, N. J. Mitra, D. Cohen-Or, Reciprocal frame structures made easy, ACM Transactions on Graphics 32 (4) (2013) 94. doi:https://doi. org/10.1145/2461912.2461915.

[15] C. Douthe, O. Baverel, Morphological and mechanical investigation of double-layer reciprocal structures, Nexus Network Journal 16 (1) (2014) 191-206. doi :https://doi.org/10.1007/ s00004-014-0185-9.

[16] D. Piker, Kangaroo: form finding with computational physics, Architectural Design 83 (2) (2013) 136-137. doi:https://doi. org/10.1002/ad.1569.

[17] T. Kohlhammer, T. Kotnik, Systemic behaviour of plane reciprocal frame structures, Structural Engineering International 21 (1) (2011) 80-86. doi:https://doi.org/10.2749/ 101686611 X12910257102596.

[18] T. Kohlhammer, A. A. Apolinarska, F. Gramazio, M. Kohler, Design and structural analysis of complex timber structures with glued t-joint connections for robotic assembly, International Journal of Space Structures 32 (3-4) (2017) 199-215. doi:https://doi.org/10.1177/0266351117746268.
[19] L. Greco, A. Lebée, C. Douthe, Investigation of the elastic behavior of reciprocal systems using homogenization techniques, in: 2013 IASS Annual Symposium: Beyond the Limit of Man, 2013, pp. 9-p.

[20] M. Brocato, Reciprocal frames: Kinematical determinacy and limit analysis, International Journal of Space Structures 26 (4) (2011) 343-358. doi:10.1260/0266-3511.26.4.343.

[21] M. Brocato, L. Mondardini, Parametric analysis of structures from flat vaults to reciprocal grids, International Journal of Solids and Structures 54 (Supplement C) (2015) $50-65$. doi:https://doi.org/10.1016/j.ijsolstr.2014.11.007.

[22] R. Poranne, R. Chen, C. Gotsman, On linear spaces of polyhedral meshes, IEEE transactions on visualization and computer graphics 21 (5) (2015) 652-662. doi:https://doi.org/ 10.1109/TVCG.2014.2388205.

[23] R. Mesnil, C. Douthe, O. Baverel, Marionette meshes: from descriptive geometry to fabrication-aware design, Advances in Architectural Geometrydoi:https://www.doi.org/10.3218/ 3778-4_7.

[24] R. Mesnil, O. Baverel, C. Douthe, Marionette meshes: modelling free-form architecture with planar facets, International Journal of Space Structures 32. doi:https://doi.org/10. 1177/0266351117738379.

[25] R. Mesnil, Structural exploration of fabrication-aware design spaces for non-standard architecture, Ph.D. thesis, Université Paris-Est (2017).

[26] R. Mesnil, O. Baverel, C. Douthe, Non-standard patterns for gridshell structures: fabrication and structural optimization, Journal of the International Association for Shell and Spatial Structures 58 (4). doi:https ://doi.org/10.20898/j.iass . 2017.194 .893

[27] S. Gelez, V. Saby, Workshop ateliers design:nexorades, facing an emergency situation, International Journal of Space Structures 26 (4) (2011) 359-361. doi:https://doi.org/10.1260/ $0266-3511.26 .4 .359$

[28] O. Popovic Larsen, Reciprocal frame (rf) structures: Real and exploratory, Nexus Network Journal 16 (1) (2014) 119-134. doi: 10.1007/s00004-014-0181-0.

[29] S. Gelez, S. Aubry, B. Vaudeville, Nexorade or reciprocal frame system applied to the design and construction of a $850 \mathrm{~m} 2$ archaeological shelter, International Journal of Space Structures 26 (4) (2011) 303-311. doi:https://doi.org/10.1260/ 0266-3511.26.4.303.

[30] H. Pottmann, M. Eigensatz, A. Vaxman, J. Wallner, Architectural geometry, Computers \& graphics 47 (2015) 145-164. doi:https://doi.org/10.1016/j.cag.2014.11.002.

[31] C. Douthe, R. Mesnil, H. Orts, O. Baverel, Isoradial meshes: Covering elastic gridshells with planar facets, Automation in Construction 83 (2017) 222-236. doi:https://doi.org/10. 1016/j.autcon.2017.08.015.

[32] A. Vaxman, A projective framework for polyhedral mesh modelling, in: Computer Graphics Forum, Vol. 33, Wiley Online Library, 2014, pp. 121-131. doi:https://doi.org/10.1111/cgf. 12405.

[33] D. Piker, W. Pearson, Plankton (2017).

[34] H. Pottmann, Y. Liu, J. Wallner, A. Bobenko, W. Wang, Geometry of multi-layer freeform structures for architecture, ACM Transactions on Graphics 26 (3) (2007) 65. doi:https: //dx.doi.org/10.1145/1275808.1276458.

[35] C. Rogers, W. Schief, On the equilibrium of shell membranes under normal loading. hidden integrability, in: Proceedings of the Royal Society of London A: Mathematical, Physical and Engineering Sciences, Vol. 459, The Royal Society, 2003, pp. 2449-2462. doi:https://doi.org/10.1098/rspa.2003.1135.

[36] C. Rüegg, M. Cuda, J. Gael, Math .NET Numerics (2012). 
[37] J. Willmann, M. Knauss, T. Bonwetsch, A. A. Apolinarska, F. Gramazio, M. Kohler, Robotic timber construction expanding additive fabrication to new dimensions, Automation in Construction 61 (Supplement C) (2016) 16 - 23. doi :https : //doi.org/10.1016/j.autcon.2015.09.011.

[38] D. Mousanezhad, B. Haghpanah, R. Ghosh, A. M. Hamouda, H. Nayeb-Hashemi, A. Vaziri, Elastic properties of chiral, antichiral, and hierarchical honeycombs: A simple energy-based approach, Theoretical and Applied Mechanics Letters 6 (2) (2016) 81 - 96. doi:https://doi.org/10.1016/j.taml.2016.02.004.

[39] J. Schlaich, H. Schober, Glass Roof for the Hippo Zoo at Berlin, Structural Engineering International 7 (4) (1997) 252-254. doi : https : //doi .org/10.2749/101686697780494581.

[40] Y. Liu, W. Wang, H. Pottmann, J. Wallner, Y. Yong-Liang, Geometric Modeling with Conical Meshes and Developable Surfaces, ACM Transactions on Graphics 25 (3) (2006) 681-689. doi:https://doi .org/10.1145/1179352.1141941.

[41] R. Mesnil, C. Douthe, O. Baverel, B. Léger, Generalised cyclidic nets for shape modelling in architecture, International Journal of Architectural Computing 15 (2) (2017) 148-168. doi :https: //doi.org/10.1177/1478077117714917.

[42] R. Mesnil, C. Douthe, O. Baverel, B. Léger, Linear buckling of quadrangular and kagome gridshells: A comparative assessment, Engineering Structures 132. doi:https://doi.org/10. 1016/j.engstruct.2016.11.039.

\section{Appendix A. Solution of the optimisation problem}

\section{Appendix A.1. Derivation of the solution}

Equation (26) can be written as an unconstrained optimisation problem with a simple substitution:

$$
\min \lambda \mathcal{F}_{L}\left(\mathbf{N N}_{\mathbf{2}} \mathbf{T}^{\prime \prime}\right)+(1-\lambda) \mathcal{F}_{E}\left(\mathbf{N N}_{\mathbf{2}} \mathbf{T}^{\prime \prime}\right)
$$

The minimum is found by computing the gradient of $\mathcal{F}_{\lambda}$ :

$$
\nabla \mathcal{F}_{\lambda}=\frac{\partial \mathcal{F}_{\lambda}}{\partial \mathbf{T}^{\prime \prime}}=\mathbf{0}
$$

We introduce additional notations:

$$
\left\{\begin{array}{lr}
\mathbf{A}_{\mathbf{E}}= & \mathbf{N}_{\mathbf{2}}{ }^{T} \mathbf{N}^{T} \mathbf{M}_{\mathbf{E}}{ }^{T} \mathbf{M}_{\mathbf{E}} \mathbf{N} \mathbf{N}_{\mathbf{2}} \\
\mathbf{A}_{\mathbf{L}}= & \mathbf{N}_{\mathbf{2}}{ }^{T} \mathbf{N}^{T} \mathbf{M}_{\mathbf{L}}{ }^{T} \mathbf{M}_{\mathbf{L}} \mathbf{N N}_{\mathbf{2}} \\
\mathbf{B}_{\mathbf{E}}= & \mathbf{E}_{\mathbf{t}}^{T} \mathbf{M}_{\mathbf{E}} \mathbf{N N}_{\mathbf{2}} \\
\mathbf{B}_{\mathbf{L}}= & \mathbf{L}_{\mathbf{t}}^{T} \mathbf{M}_{\mathbf{L}} \mathbf{N N}_{\mathbf{2}}
\end{array}\right.
$$

and

$$
\left\{\begin{array}{l}
\mathbf{A}_{\lambda}=(1-\lambda) \mathbf{A}_{\mathbf{L}}+\lambda \mathbf{A}_{\mathbf{E}} \\
\mathbf{B}_{\lambda}=(1-\lambda) \mathbf{B}_{\mathbf{L}}+\lambda \mathbf{B}_{\mathbf{E}}
\end{array}\right.
$$

The gradient is computed with elementary rules of matrix calculus:

$$
\nabla \partial \mathcal{F}_{\lambda}=2 \mathbf{A}_{\lambda} \mathbf{T}^{\prime \prime}-2 \mathbf{B}_{\lambda}
$$

The nullity of the gradient implies that the translations are solution of a linear system of equations:

$$
\mathbf{A}_{\lambda} \mathbf{T}^{\prime \prime}=\mathbf{B}_{\lambda}
$$

This system can be solved using various decompositions. We used Cholesky decomposition, which only works on symmetric definite positive matrices. The matrix $\mathbf{A}_{\lambda}$ is obviously symmetric, and the numerous examples treated in this paper tend to suggest that it is symmetric positive. The translations are then found by applying the substitution $\mathbf{T}=\mathbf{N N}_{\mathbf{2}} \mathbf{T}^{\prime \prime}$.

\section{Appendix A.2. Dependency of the solution on $\alpha$}

We study more in detail the subcase $\mathbf{E}_{\mathbf{t}}=\mathbf{0}$ and $\mathbf{L}_{\mathbf{t}}=$ $\alpha L_{T} \mathbf{1}$, which is treated in the examples of this article. In this case, it is clear that $\mathbf{B}_{\mathbf{E}}=\mathbf{0}$, and that, in consequence $\mathbf{B}_{\lambda}=(1-\lambda) \mathbf{B}_{\mathbf{L}}$. Equation A.4 can be re-written as follows:

$$
\mathbf{A}_{\lambda} \mathbf{T}^{\prime \prime}=(1-\lambda) \cdot \alpha \cdot L_{T} \cdot \mathbf{1}^{T} \mathbf{M}_{\mathbf{L}} \mathbf{N N}_{\mathbf{2}}
$$

It appears thus that the translation depends linearly on $\alpha$. Since the engagement lengths and eccentricities depend linearly on the translations, it means that they also depend linearly on $\alpha$. 